\title{
Aplicação superficial de escória, lama cal, lodos de esgoto e calcário na cultura da soja
}

\author{
Juliano Corulli Corrêa $a^{(1)}$, Leonardo Theodoro Büll(1), Carlos Alexandre Costa Crusciol(1) \\ e Marco Antonio Tecchio(2)
}

(1)Universidade Estadual Paulista, Faculdade de Ciências Agronômicas, Fazenda Experimental Lageado, Caixa Postal 237, CEP 18603-970
Botucatu, SP. E-mail: correajc@superig.com.br, bull@fca.unesp.br, crusciol@fca.unesp.br (2)Instituto Agronômico de Campinas, Centro
Avançado de Pesquisa Tecnológica do Agronegócio de Frutas, Caixa Postal 11, CEP 13214-820, Jundiaí, SP. E-mail: tecchio@iac.sp.gov.br

Resumo - O objetivo deste trabalho foi avaliar o efeito da aplicação superficial de lodos de esgoto, lama cal, escória de aciaria e calcário sobre o estado nutricional e a produtividade da soja, em sistema plantio direto. $\mathrm{O}$ delineamento foi o de blocos ao acaso em arranjo fatorial $4 \times 4+1$, constituído por quatro tratamentos resíduos de lodo de esgoto centrifugado (LC) e de biodigestor (LB), escória de aciaria (E) e lama cal (Lcal) - nas doses 0, 2, 4 e $8 \mathrm{Mg} \mathrm{ha}^{-1}$, mais o controle com $2 \mathrm{Mg} \mathrm{ha}^{-1}$ de calcário. As plantas de soja apresentaram maior concentração de nitrogênio, fósforo e cálcio, em 2003, 2004 e 2005, e de potássio, em 2003 e 2004, em razão dos tratamentos LC, LB, E, Lcal e calagem. A produtividade da soja foi favorecida pela aplicação dos tratamentos no sistema plantio direto, em 2003, 2004 e 2005. O fósforo, e o cálcio contribuíram para o aumento da produtividade da soja em 2003 e 2004.

Termos para indexação: Glycine max, concentração de nutrientes, conservacão do solo, reciclagem de resíduos.

\section{Surface application of flue dust, aqueous lime, sewage sludge and limestone on soybean crop}

\begin{abstract}
The objective of this work was to evaluate the effect of the surface application of sewage sludge, aqueous lime, flue dust and limestone on soybean nutrition and yield in no-till system. The experiment was arranged in a randomized complete block design, in factorial scheme of $4 \times 4+1$, and consisted of four residues: centrifuged sewage sludge (CSS), biodigestor sewage sludge (BSS), flue dust (FD) and aqueous lime (AL), at $0,2,4$ and $8 \mathrm{Mg} \mathrm{ha}^{-1}$, and one additional control treatment with dolomitic limestone at $2 \mathrm{Mg} \mathrm{ha}^{-1}$. The soybean plants showed greater contents of nitrogen, phosphorus and calcium in 2003, 2004 and 2005, and potassium contents in 2003 and 2004, due to the treatments CSS, BSS, FD, AL and limestone. Soybean grain yield was also enhanced due to surface applications of the treatments in no-till system in 2003, 2004 and 2005. The phosphorus and calcium contributed to increase soybean yield in 2003 and 2004.
\end{abstract}

Index terms: Glycine max, nutrient concentration, soil conservation, waste recycling.

\section{Introdução}

A utilização de resíduos industriais e lodos de esgoto na agricultura pode contribuir para o aproveitamento, pelas plantas, dos elementos essenciais e compostos orgânicos neles contidos (Oliveira et al., 2002, Nascimento et al., 2004), e favorecer ao desenvolvimento e à produtividade das espécies vegetais cultivadas (Carvalho-Pupatto et al., 2004; Vieira et al., 2005; Lemainski \& Silva, 2006). Essa prática inspira cuidados, pois se não atender aos padrões existentes na legislação pode ocorrer aumento dos teores de metais pesados, nitrato e fósforo no solo, bem como a possível transmissão de organismos patogênicos ao homem e aos animais.
Trabalhos de pesquisa, que demonstram os benefícios da aplicação de resíduos no solo para cultura da soja, tratam da condição de incorporação desses resíduos, e entre eles o de Lemainski \& Silva (2006), que observaram a viabilidade agronômica e econômica do uso do lodo de esgoto em substituição ao fertilizante mineral. Vieira (2001) e Vieira et al. (2005) verificaram que a necessidade de $\mathrm{P}$ da cultura soja pode ser suprida pela adição de lodo de esgoto ao solo.

No sistema plantio direto, já implantado, evita-se a incorporação de corretivos, a fim de preservar as propriedades químicas, físicas e biológicas do solo, então, opta-se por fazer a aplicação sobre a superfície do solo. Com base nesse princípio, Corrêa et al. (2007, 2008) avaliaram a aplicação superficial de lodos de 
esgoto, escória de aciaria e lama cal, e demonstraram que essa prática proporciona a neutralização da acidez, eleva a saturação por bases e aumenta a disponibilidade de nitrato, cálcio e magnésio até a profundidade de $40 \mathrm{~cm}$ do solo, e não disponibiliza metais pesados ao meio ambiente. A aplicação de lodos de esgoto sobre a superfície do solo - seguida ou não de incorporação -

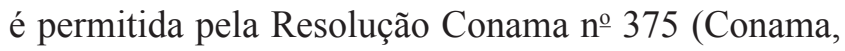
2006).

Espera-se que a aplicação superficial de escória de aciaria, lama cal e lodos de esgoto, no sistema plantio direto, ao favorecer a fertilidade do solo, possa aumentar a quantidade de nutrientes absorvidos pela soja, os quais serão utilizados para o seu crescimento e desenvolvimento (Rossetto et al., 1995). Os mesmos benefícios atribuídos à aplicação superficial de calcário no sistema plantio direto podem ser atribuídos à aplicação desses resíduos, uma vez que esse sistema de produção permite dinâmica diferenciada dos atributos de acidez decorrente do acúmulo superficial de matéria orgânica e de nutrientes, menor toxidez por $\mathrm{Al}$ e maior disponibilidade de água (Oliveira \& Pavan, 1996, Caires et al., 2003).

O objetivo do trabalho foi avaliar o efeito da aplicação superficial de lodos de esgoto, lama cal e escória de aciaria sobre o estado nutricional e a produtividade da soja no sistema plantio direto.

\section{Material e Métodos}

O experimento foi conduzido nos anos agrícolas de 2002 a 2005, na Fazenda Experimental Lageado, pertencente à Faculdade de Ciências Agronômicas da Unesp, Botucatu, SP, a 22 $51^{\prime} 15^{\prime \prime} \mathrm{S}$, e $48^{\circ} 26^{\prime} 30^{\prime \prime} \mathrm{W}$ e altitude de $740 \mathrm{~m}$, com declividade menor que $10 \%$. O clima predominante é do tipo $\mathrm{Cwb}$ segundo a classificação de Köppen.

$\mathrm{O}$ experimento foi implantado em Latossolo Vermelho distrófico, textura média, fase campo subtropical, relevo suave ondulado (Embrapa, 1999) e manejado sob sistema plantio direto. A amostragem para a caracterização química do solo foi realizada na camada de 0-20 cm, e apresentou: $\mathrm{pH}\left(\mathrm{CaCl}_{2}\right), 4,1$; $43 \mathrm{mmol}_{\mathrm{c}} \mathrm{dm}^{-3}$ de $\mathrm{H}+\mathrm{Al}$ (Quaggio \& Raij, 2001); $16 \mathrm{~g} \mathrm{~kg}^{-1}$ de MO (Cantarella et al., 2001); 5,6 mg dm${ }^{-3}$ de P (resina); 0,8, 15 e $6 \mathrm{mmol}_{\mathrm{c}} \mathrm{dm}^{-3}$ de $\mathrm{K}, \mathrm{Ca}$ e $\mathrm{Mg}$, respectivamente (Raij \& Quaggio, 2001) e 34\% de saturação por bases. As amostragens de solo, para determinação do $\mathrm{pH}$ em $\mathrm{CaCl}_{2}, \mathrm{P}$ (resina), $\mathrm{Ca}$ e $\mathrm{Mg}$ no solo, também foram realizadas na camada de 0-20 cm, onde ocorre maior exploração radicular e maior absorção de nutrientes. Foi utilizando trado do tipo sonda, e feita a coleta de quatro amostras simples para compor a amostra composta de cada parcela, sendo retiradas antes da semeadura da soja e seguindo a metodologia anterior.

O delineamento em blocos ao acaso com quatro repetições, em arranjo fatorial $4 \times 4+1$, foi constituído por quatro resíduos: LC, lodo de esgoto centrifugado; LB, lodo de esgoto de biodigestor; E, escória de aciaria e Lcal, lama cal, nas doses de 0, 2, 4 e $8 \mathrm{Mg} \mathrm{ha}^{-1}$, em base seca, mais um controle de $2 \mathrm{Mg} \mathrm{ha}^{-1}$ de calcário, todos aplicados na superfície do solo. Em cada bloco, as parcelas foram dimensionadas com $6 \mathrm{~m}$ de largura por $7 \mathrm{~m}$ de comprimento.

$\mathrm{O}$ tratamento-controle correspondeu à aplicação de calcário dolomítico $\left(280 \mathrm{~g} \mathrm{~kg}^{-1}\right.$ de $\mathrm{CaO}, 230 \mathrm{~g} \mathrm{~kg}^{-1}$ de $\mathrm{MgO}$ e PRNT de 70\%) na dose recomendada pelo método de saturação por bases $\left(2 \mathrm{Mg} \mathrm{ha}^{-1}\right)$ para elevar este índice a $70 \%$, com base de cálculo para a camada de $0-20 \mathrm{~cm}$.

O lodo de esgoto centrifugado foi obtido na estação de tratamento de esgoto (ETE) de São José dos Campos, o qual recebeu tratamento com óxido de cálcio para a eliminação de organismos patogênicos e foi classificado como de classe A; já o lodo de esgoto de biodigestor foi obtido na ETE de Barueri, e recebeu a adição de polieletrólitos, e foi classificado como de classe B. Ambos os lodos passaram pelo processo de solarização, com utilização de lona de plástico transparente, para a redução do teor de umidade e possível redução de organismos patogênicos, antes de serem aplicados ao solo. A escória de aciaria foi proveniente da Empresa Mannesmann, e é considerada resíduo da indústria da fundição do aço e do ferro-gusa. A lama cal, proveniente da Empresa Luwarcel, é considerada resíduo da fabricação de papel e celulose, e foi gerada no processo de clarificação da celulose. O lodo de esgoto de biodigestor é o único resíduo que não pode ser considerado corretivo de acidez, no entanto, alguns trabalhos têm demonstrado o aumento do pH do solo em razão da formação de íons amônio, devido à oxidação do $\mathrm{N}$ orgânico (Berton et al., 1989).

Para a escolha das doses dos resíduos foi montado um experimento preliminar de incubação, em sacolas de plásticos de $0,5 \mathrm{~kg}$ de solo, com doses de 1 a 
$20 \mathrm{Mg} \mathrm{ha}^{-1}$, para cada resíduo, e tendo como critério a recomendação de não ultrapassar o valor de $\mathrm{pH} 7$ em $\mathrm{CaCl}_{2}$. Observou-se que a lama cal alcançou o $\mathrm{pH} 7$, no solo, quando esse se encontrava com $70 \%$ de capacidade de campo, na dose de $8 \mathrm{Mg} \mathrm{ha}^{-1}$. Após dois meses de incubação, procurou-se adotar valores abaixo dessa dose, selecionando os números que pudessem formar uma progressão geométrica, para melhor ajuste do comportamento matemático das variáveis. Mesmo sabendo que existia diferença de propriedades químicas entre os resíduos, optou-se por utilizar doses semelhantes em razão da estatística experimental.

Antes da instalação do experimento, determinaramse a análise química e o teor total de metais pesados presentes em cada resíduo (Tabela 1). Utilizou-se a metodologia do Lanarv (1983), com base nos ácidos nítrico e clorídrico, os quais apresentam concentrações ainda maiores que os da EPA SW-846, porém sem a presença da água oxigenada (Abreu et al., 2001). As leituras foram analisadas em espectrofotômetro de emissão em plasma induzido em argônio (ICP/AES). Os teores de metais pesados, encontrados nos resíduos industriais Lcal e E, foram inferiores às quantidades permitidas na NBR 10004 (ABNT, 1987), e os teores de metais pesados dos lodos de esgoto - LB e LC foram inferiores ao permitido pela Resolução Conama no 375 (Conama, 2006). Foram determinados a reatividade $(\mathrm{RE})$, o poder neutralizante $(\mathrm{PN})$ e o poder relativo de neutralização total (PRNT) da lama cal, dos lodos de esgoto (LC e LB), escória de aciaria e calcário (Tabela 2), pelo método do Lanarv (1983) (Tabela 2).

Antes da implantação do experimento a área experimental era cultivada em sistema plantio direto, com a seqüência de culturas da soja, aveia, milho e um ano em pousio. Em 2002, iniciou-se o experimento com a dessecação do feijão guandu (Cajanus cajan L.), semeado para produzir palhada. Essa espécie de cobertura vegetal produziu $7,4 \mathrm{Mg} \mathrm{ha}^{-1}$ de massa de matéria seca e teores de $8 \mathrm{~g} \mathrm{~kg}^{-1}$ de Ca, $3,2 \mathrm{~g} \mathrm{~kg}^{-1}$ de $\mathrm{Mg}, 13 \mathrm{~g} \mathrm{~kg}^{-1}$ de $\mathrm{N}$ e 1,6 $\mathrm{g} \mathrm{kg}^{-1}$ de $\mathrm{S}$. Em seguida, os tratamentos foram aplicados a lanço sobre a palhada do guandu, sem incorporação, em agosto de 2002. A aplicação antecedeu três meses à semeadura da soja, na safra 2002/2003. A precipitação pluvial nesse período foi de $270 \mathrm{~mm}$. De novembro de 2002 a maio de 2005 a soma do volume de precipitação atingiu $2.850 \mathrm{~mm}$.

Durante o período do experimento foi realizada a semeadura da cultivar de soja EMBRAPA 48, submetida à inoculação de Bradyrhizobium japonicum, nas safras de 2002/2003, 2003/2004 e 2004/2005. Optou-se por repetir a mesma cultura de soja, no verão, e a aveiapreta, no inverno, nas safras de 2003 e 2004, para que houvesse maior repetição dos resultados em razão da aplicação superficial de doses crescentes dos resíduos, o que permitiu maior confiabilidade nos dados, principalmente, por se tratar de experimento em

Tabela 1. Caracterização química dos resíduos de lodo de esgoto centrifugado (LC) e de biodigestor (LB), escória de aciaria (E) e lama cal (Lcal) e comparação dos limites de metais pesados com a legislação em vigor.

\begin{tabular}{|c|c|c|c|c|c|c|c|c|c|c|c|c|c|}
\hline Resíduos & Umidade & $\mathrm{pH}$ & $\mathrm{K}$ & $\mathrm{N}$ & $\mathrm{Ca}$ & $\mathrm{Mg}$ & $\mathrm{P}$ & As & $\mathrm{Cd}$ & $\mathrm{Cr}$ & $\mathrm{Hg}$ & $\mathrm{Ni}$ & $\mathrm{Pb}$ \\
\hline & $\left(\mathrm{g} \mathrm{kg}^{-1}\right)$ & & ------ & (g 1 & $\mathrm{e} \mathrm{ma}$ & eca) & -1- & -----. & - & $\mathrm{g} \mathrm{kg}^{-1} \mathrm{c}$ & téria & -.- & 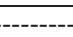 \\
\hline $\mathrm{LC}$ & 50 & 12 & 2,5 & 25 & 280 & 4 & 2,2 & 14 & 0,0 & 4 & 0 & 7 & 17 \\
\hline LB & 290 & 7 & 1,7 & 40 & 20 & 4 & 7,0 & 27 & 0,1 & 19 & 0 & 180 & 107 \\
\hline E & 20 & 12 & 0,8 & 3 & 230 & 21 & 0,0 & 5 & 0,0 & 61 & 0 & 19 & 308 \\
\hline Lcal & 190 & 12 & 0,8 & 4 & 370 & 6 & 1,7 & 1,4 & 0,0 & 13 & 0 & 96 & 60 \\
\hline$\overline{\text { Resolução }}^{(1)}$ & & & & & & & & 41 & 39 & 1.000 & 17 & 420 & 300 \\
\hline Normas $^{(2)}$ & & & & & & & & 1.000 & n. $\mathrm{a}^{(3)}$ & n.a & 100 & n.a & 1.000 \\
\hline
\end{tabular}

${ }^{(1)}$ Legislação para os padrões legais vigentes de metais pesados para lodos de esgoto: Resolução Conama no 387 (Conama, 2006 ). ${ }^{(2)}$ Legislação para os padrões legais vigentes de metais pesados para resíduos sólidos: NBR 10004 (ABNT, 1987). (3)Não aplicável (não descrito o limite na NBR 10004).

Tabela 2. Percentagem de partículas retidas nas peneiras de 10, 20, 50 e fundo e reatividade (RE), poder neutralizante (PN) e poder relativo de neutralização total (PRNT), dos resíduos de lodo de esgoto centrifugado (LC) e de biodigestor (LB), escória de aciaria (E), lama cal (Lcal) e calcário.

\begin{tabular}{|c|c|c|c|c|c|c|c|}
\hline Resíduos & $\begin{array}{c}\text { Pen } 10 \\
\varnothing=2 \mathrm{~mm} \\
\end{array}$ & $\begin{array}{c}\text { Pen } 20 \\
\varnothing=0,8 \mathrm{~mm}\end{array}$ & $\begin{array}{c}\text { Pen } 50 \\
\varnothing=0,3 \mathrm{~mm}\end{array}$ & Fundo & $\mathrm{RE}$ & $\mathrm{PN}$ & PRNT \\
\hline$\overline{\mathrm{LC}}$ & 2,4 & 14,6 & 40,1 & 42,9 & 69,8 & 93,0 & 65 \\
\hline LB & 0,1 & 50,2 & 35,5 & 14,2 & 36,5 & 3,0 & 1,1 \\
\hline E & 2,2 & 6,1 & 32,5 & 59,1 & 79,8 & 60,0 & 47 \\
\hline Lcal & 0,0 & 0,0 & 0,1 & 99,9 & 99,9 & 99,0 & 98 \\
\hline Calcário & 0,0 & 4,2 & 18,8 & 77,0 & 89,1 & 80,0 & 71 \\
\hline
\end{tabular}


campo, bem como recomendar a sucessão gramíneas/ leguminosas, um dos pré-requisitos do sistema plantio direto.

Para a cultura da soja, a adubação de base anual no sulco da semeadura foi de $60 \mathrm{~kg} \mathrm{ha}^{-1}$ de $\mathrm{P}_{2} \mathrm{O}_{5}$ e $30 \mathrm{~kg} \mathrm{ha}^{-1}$ de $\mathrm{K}_{2} \mathrm{O}$ do formulado 0-20-10; e para a aveia-preta, foi de $46 \mathrm{~kg} \mathrm{ha}^{-1}$ de $\mathrm{N}$, na forma de uréia, a fim de melhorar ainda mais a produção de biomassa vegetal para formação de palhada e, conseqüentemente, aumentar os benefícios proporcionados nos atributos químicos, físicos e biológicos do solo, e favorecer, de forma indireta, as reações dos resíduos aplicados sobre a superfície do solo.

Para a avaliação dos teores de N, P, K, Ca e Mg, na cultura da soja, foram amostrados 50 trifólios com pecíolo, amostrando-se o terceiro trifólio a partir do ápice, quando a planta encontrava-se no estádio R2, em pleno florescimento, sendo que a análise química do tecido vegetal seguiu a metodologia de Malavolta et al. (1997). Os valores de produtividade foram obtidos pela colheita e pesagem dos grãos, e posterior correção do peso para base seca de $13 \%$ de umidade.

Os resultados foram submetidos à análise de variância, pelo SISVAR 4.2 (Ferreira, 2003), e posterior regressão, ajustando-se as equações aos dados obtidos a partir das doses dos resíduos, adotando-se como critério para escolha do modelo, a interação pelo teste $\mathrm{F}$, significativo a $5 \%$ e magnitude dos coeficientes de determinação (Banzato \& Kronka, 1989), e os resultados foram analisados pelo Sigmaplot 8.0. Todos os tratamentos foram comparados com o calcário, com a aplicação do teste t (DMS), a 5\% de probabilidade, pelo SISVAR 4.2. Para análise de correlação, foi adotado o método de Pearson, e essa análise e a de regressão linear múltipla foram realizadas pelo SIGMASTAT 3.11 (Systat Software, 2008).

\section{Resultados e Discussão}

A aplicação de LC, LB, E e Lcal e o calcário aumentou o teor de $\mathrm{N}$ na soja, durante os três anos agrícolas (Figura 1). Esse aumento pode ser resultado da melhoria nos atributos químicos do solo (Tabela 3) como $\mathrm{pH}$ e aumento na concentração de $\mathrm{P}, \mathrm{Ca} \mathrm{e}$ $\mathrm{Mg}$, fatores esses que podem proporcionar melhor desenvolvimento radicular e, conseqüentemente, maior interação entre as bactérias do grupo Rhizobium e a raiz, o que permite maior fixação biológica de nitrogênio. Outra provável justificativa para o aumento de $\mathrm{N}$ nas folhas de soja é que alguns resíduos como os lodos (LC e LB) são fontes diretas desse nutriente no solo, sendo que LC e LB apresentam 25 e $40 \mathrm{~g} \mathrm{~kg}^{-1}$ de $\mathrm{N}$, respectivamente (Tabela 1 ).

Alcântara et al. (2007), ao trabalhar com doses crescentes de lodos de curtume, verificaram que a aplicação desse resíduo em Latossolo diminui a taxa de mineralização do $\mathrm{N}$ proveniente do lodo e aumenta substancialmente o tempo de meia-vida no solo. Esses resultados permitem inferir que doses elevadas de lodo (dependendo da sua qualidade) poderão implicar grandes liberações de $\mathrm{N}$, em médio prazo, resultado que pode interferir no desenvolvimento das plantas (e até em poluição ambiental).

No terceiro ano (2005) de cultivo da soja, os resíduos industriais, os lodos de esgoto e o calcário ainda continuam favorecendo o aumento do teor de $\mathrm{N}$, com destaque para Lcal, na dose de 5,5 $\mathrm{Mg} \mathrm{ha}^{-1}$, e E e LC na dose de $8 \mathrm{Mg} \mathrm{ha}^{-1}$ (Figura 1). Esse incremento no teor de $\mathrm{N}$ nas plantas de soja corresponderam em aumento de até $15 \mathrm{~g} \mathrm{~kg}^{-1}$, para os melhores tratamentos no ano de 2005. O maior fornecimento desse nutriente ao solo também está relacionado à maior reciclagem no solo pelo sistema plantio direto, fato atribuído ao aumento de biomassa seca da parte aérea de aveia preta nos anos de 2003 e 2004, alcançando valores de $6,4,6,0,5,7,5,0$ e $5,1 \mathrm{Mg} \mathrm{ha}^{-1}$, para os tratamentos com E, Lcal, LC, LB e calcário, no ano de 2003 e valores de 5,5, 5,9, 5,4,5,4 e 4,6 $\mathrm{Mg} \mathrm{ha}^{-1}$, no ano de 2004, sendo que esses melhores resultados conferiram o aporte de 134, 132, 125, 120 e $102 \mathrm{~kg} \mathrm{ha}^{-1}$ de N, no ano de 2003 e de $83,88,81,92$ e $73 \mathrm{~kg} \mathrm{ha}^{-1}$ de $\mathrm{N}$ no ano de 2004.

Para Malavolta et al. (1999), a concentração adequada de $\mathrm{N}$ na folha, para uma produtividade de 2,7 $\mathrm{Mg} \mathrm{ha}^{-1}$ de soja, seria de $36 \mathrm{~g} \mathrm{~kg}^{-1}$. Em 2003 e 2004, os resultados dos tratamentos com resíduos e calcário ficaram acima do estabelecido para a produtividade de soja, e apenas a dose 0 e os tratamentos com 2 e $4 \mathrm{Mg} \mathrm{ha}^{-1}$ de LB estiveram abaixo de $36 \mathrm{~g} \mathrm{~kg}^{-1}$ no ano de 2005 (Figura 1).

A concentração de $\mathrm{N}$ nas folhas de soja diminuiu do primeiro para o terceiro cultivo, o que é indicativo de que a quantidade de $\mathrm{N}$ presente nos resíduos aplicados foi disponibilizada às plantas de soja no primeiro e segundo anos de cultivo (Figura 1). Esse resultado corrobora os de Alcântara et al. (2007). 

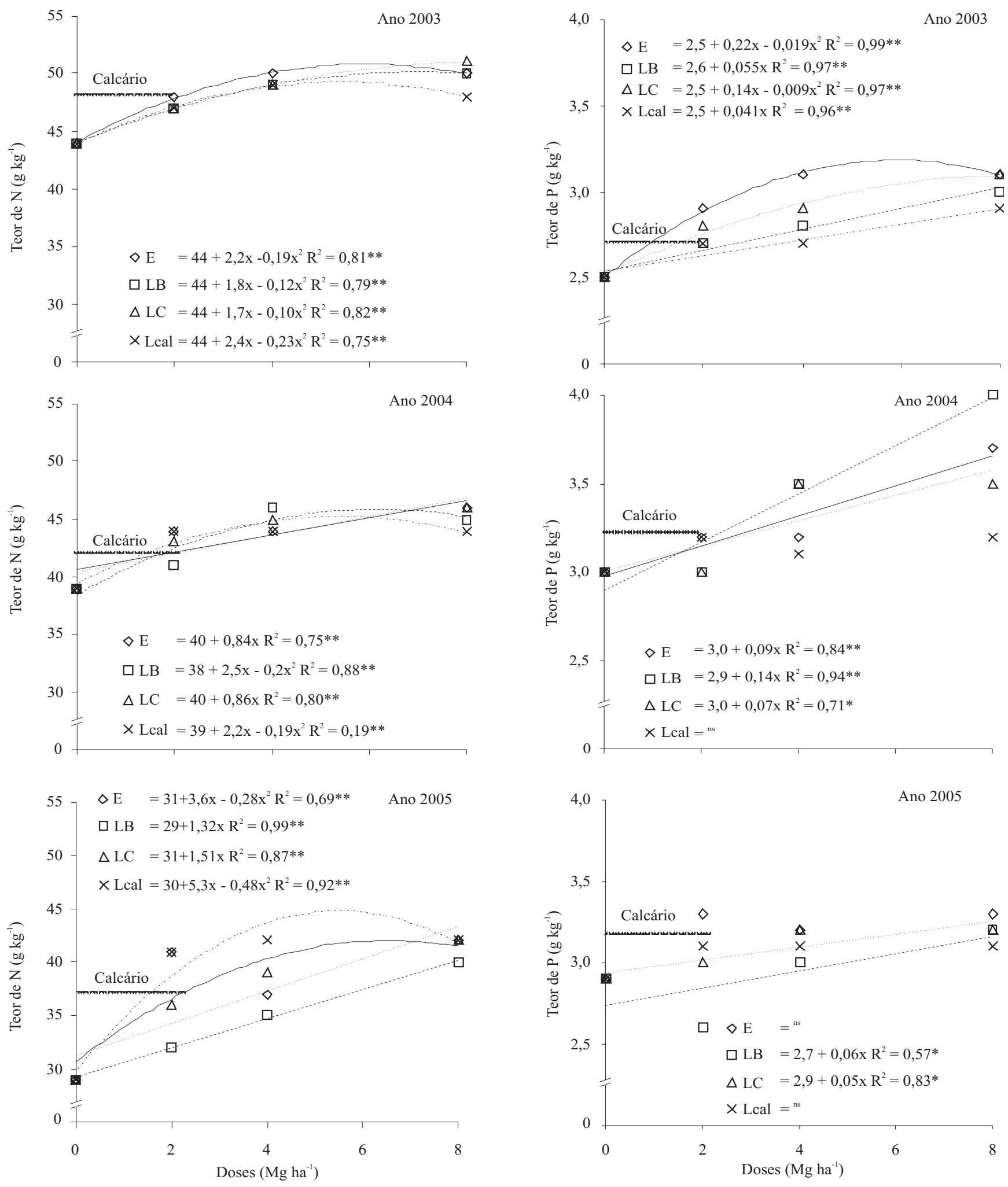

Figura 1. Teores de nitrogênio e fósforo nas folhas de soja, nos anos de 2003, 2004 e 2005, em razão da aplicação de resíduos de lodo de esgoto centrifugado (LC) e de biodigestor (LB), escória de aciaria (E), lama cal (Lcal) e calcário na superfície do solo em sistema plantio direto. 
O teor de P na soja foi aumentado em conseqüência da aplicação dos resíduos e do calcário (Figura 1), com exceção do tratamento Lcal, nos anos de 2004 e 2005, e do tratamento E no ano de 2005. Esse resultado pode ser atribuído ao fato de os tratamentos LC, LB, E e Lcal terem fornecido o total de:160, 392, 112 e $16 \mathrm{~kg} \mathrm{ha}^{-1}$ de $\mathrm{P}_{2} \mathrm{O}_{5}$, respectivamente, em suas maiores doses, bem como ao aumento nos valores de $\mathrm{pH}$ do solo nos tratamentos LC, E, Lcal e calcário (Tabela 3), fato esse que auxiliou a maior disponibilidade de $\mathrm{P}$ para absorção das plantas. A contribuição da calagem no teor de P nas folhas de soja diminuiu com o tempo, e foi inferior aos resultados encontrados nos resíduos, em especial, na dose de $8 \mathrm{Mg} \mathrm{ha}^{-1}$.

O teor de 2,6 $\mathrm{g} \mathrm{kg}^{-1}$ de P na folha de soja é considerado adequado para se obter uma produtividade de $3 \mathrm{Mg} \mathrm{ha}^{-1}$ (Malavolta et al., 1999). Todos os tratamentos apresentaram valores de $\mathrm{P}$ maiores, com exceção para a dose 0 , no primeiro ano de cultivo. A adição de lodo de esgoto ao solo tem aumentado a disponibilidade de P para diversas culturas, entre elas a soja (Brown et al., 1996; Vieira, 2001; Lemainski \& Silva, 2006). Mesmo com aumentos significativos nos teores de $\mathrm{P}$ e $\mathrm{N}$ no solo, pela adição de lodo de esgoto, Nascimento et al. (2004) relatam que esse resíduo apresenta concentração baixa de $\mathrm{P}$, havendo necessidade de suplementação com fertilizantes minerais para a obtenção de altas produtividades.
Na soja, os teores de K aumentaram com a aplicação dos resíduos E e Lcal no ano de 2003; já em 2004, todos os resíduos favoreceram à absorção desse nutriente, obtendo-se comportamento linear com E e LC e quadrático com LB e Lcal (Figura 2). Em 2005, não foram verificadas diferenças para o teor de $\mathrm{K}$ nas folhas de soja, resultado justificado pelo tratamento sem resíduo ter apresentado teor de 3,2 $\mathrm{mmol}_{\mathrm{c}} \mathrm{dm}^{-3}$ de K no solo. Esse valor é considerado alto para fertilidade do solo (Raij et al., 1996), na camada de 0-20 cm, o que pode favorecer o aumento do transporte por fluxo de massa em detrimento à difusão (Rosolem et al., 2003), uma vez que o $\mathrm{K}$ apresenta alta mobilidade no solo (Oliveira et al., 2001). Além disso, as plantas apresentam mecanismo duplo de absorção para $\mathrm{K}$, isso de forma ativa, pelas proteínas transportadoras e de forma passiva pelas proteínas canais, quando os teores de $\mathrm{K}$ se encontram com baixas e altas concentrações no solo (Malavolta, 2004), e isso permitiu que a soja, em 2005, apresentasse alto consumo para K, em razão da elevada concentração no solo, mesmo no tratamento em que não ocorreu a aplicação superficial de resíduos e calcário.

Em 2003 e 2004, houve maior concentração de Ca nas folhas de soja em razão do aumento das doses dos resíduos e da calagem, com exceção de LB, em 2004 (Figura 3). O aumento do teor de Ca na soja é justificado pelo fato de os resíduos LC, LB, E e Lcal

Tabela 3. Atributos químicos do solo em razão da aplicação superficial de lodo de esgoto centrifugado (LC) e de biodigestor (LB), escória de aciaria (E), lama cal (Lcal) e calcário sob plantio direto, 3, 15 e 27 meses após a aplicação ${ }^{(1)}$.

\begin{tabular}{|c|c|c|c|c|c|c|c|c|c|c|c|c|c|}
\hline \multirow[t]{2}{*}{ Tratamentos } & \multirow{2}{*}{$\begin{array}{c}\text { Doses } \\
\left(\mathrm{Mg} \mathrm{ha}^{-1}\right)\end{array}$} & \multicolumn{3}{|c|}{$\mathrm{pH} \mathrm{CaCl}{ }_{2}$} & \multicolumn{3}{|c|}{ P resina $\left(\mathrm{mg} \mathrm{dm}^{-3}\right)$} & \multicolumn{3}{|c|}{$\mathrm{Ca}\left(\mathrm{mmol}_{\mathrm{c}} \mathrm{dm}^{-3}\right)$} & \multicolumn{3}{|c|}{$\mathrm{Mg}\left(\mathrm{mmol}_{\mathrm{c}} \mathrm{dm}^{-3}\right)$} \\
\hline & & 3 meses & 15 meses & 27 meses & 3 meses & 15 meses & 27 meses & 3 meses & 15 meses & 27 meses & 3 meses & 15 meses & 27 meses \\
\hline $\mathrm{LC}$ & 2 & 4,5 & 4,6 & 5,0 & 13,7 & 12,7 & 17,0 & 28 & 18 & 25 & 8 & 8 & 8 \\
\hline $\mathrm{LC}$ & 4 & 4,5 & 5,2 & 5,2 & 13,1 & 36,7 & 24,3 & 28 & 48 & 33 & 8 & 8 & 8 \\
\hline LC & 8 & 5,1 & 6,6 & 6,0 & 24,1 & 63,3 & 39,0 & 45 & 89 & 60 & 9 & 9 & 9 \\
\hline Regressão & & $\mathrm{Q}^{* *}$ & $\mathrm{~L} * *$ & $\mathrm{~L}^{* *}$ & $\mathrm{~L}^{*}$ & $\mathrm{~L} * *$ & $\mathrm{Q}^{* *}$ & $\mathrm{~L}^{* *}$ & $\mathrm{~L}^{* *}$ & $\mathrm{Q}^{* *}$ & $\mathrm{Q}^{*}$ & ns & $\mathrm{Q}^{*}$ \\
\hline LB & 2 & 4,1 & 4,2 & 4,4 & 7,8 & 22,7 & 22,0 & 16 & 14 & 15 & 6 & 7 & 7 \\
\hline LB & 4 & 4,2 & 4,2 & 4,5 & 11,3 & 45,3 & 22,3 & 19 & 19 & 19 & 7 & 8 & 8 \\
\hline LB & 8 & 4,2 & 4,1 & 4,3 & 14,1 & 54,3 & 27,7 & 20 & 18 & 19 & 7 & 6 & 8 \\
\hline Regressão & & ns & $\mathrm{ns}$ & ns & $\mathrm{L}^{* *}$ & $\mathrm{~L}^{* *}$ & $\mathrm{~L} * *$ & $\mathrm{~L}^{*}$ & $\mathrm{~ns}$ & ns & ns & ns & ns \\
\hline $\mathrm{E}$ & 2 & 4,4 & 4,5 & 4,8 & 9,5 & 21,7 & 22,7 & 23 & 25 & 20 & 8 & 9 & 8 \\
\hline E & 4 & 4,7 & 4,7 & 4,9 & 15,5 & 22,0 & 25,0 & 38 & 29 & 30 & 10 & 12 & 12 \\
\hline $\mathrm{E}$ & 8 & 4,8 & 5,4 & 5,5 & 16,3 & 26,0 & 32,3 & 47 & 58 & 38 & 13 & 14 & 14 \\
\hline Regressão & & $\mathrm{L}^{* *}$ & $\mathrm{~L} * *$ & $\mathrm{~L}^{* *}$ & $\mathrm{~L}^{* *}$ & $\mathrm{Q}^{*}$ & $\mathrm{~L}^{* *}$ & $\mathrm{~L}^{* *}$ & $\mathrm{~L}^{* *}$ & $\mathrm{~L}^{* *}$ & $\mathrm{~L}^{* *}$ & $\mathrm{Q}^{*}$ & $\mathrm{~L}^{* *}$ \\
\hline Lcal & 2 & 4,3 & 5,0 & 4,3 & 6,5 & 20,3 & 19,3 & 20 & 43 & 32 & 5 & 9 & 8 \\
\hline Lcal & 4 & 4,6 & 5,4 & 4,6 & 9,7 & 19,0 & 20,0 & 26 & 49 & 37 & 6 & 8 & 9 \\
\hline Lcal & 8 & 5,1 & 5,9 & 5,0 & 14,9 & 21,3 & 23,3 & 42 & 67 & 70 & 9 & 8 & 11 \\
\hline Regressão & & $\mathrm{L}^{* *}$ & $\mathrm{~L}^{* *}$ & $\mathrm{~L}^{* *}$ & $\mathrm{~L}^{*}$ & $\mathrm{Q}^{*}$ & $\mathrm{~L}^{* *}$ & $\mathrm{~L}^{* *}$ & $\mathrm{Q}^{* *}$ & $\mathrm{~L}^{* *}$ & $\mathrm{~L}^{* *}$ & ns & $\mathrm{L}^{* *}$ \\
\hline Testemunha & 0 & 4,1 & 4,1 & 4,5 & 7,0 & 12,7 & 15,7 & 15 & 11 & 12 & 6 & 6 & 6 \\
\hline Calcário & 2 & 4,4 & 4,8 & 4,8 & 8,3 & 16,3 & 24,7 & 23 & 24 & 27 & 6 & 14 & 15 \\
\hline $\mathrm{CV}(\%)$ & & 6 & 7 & 6 & 18 & 15 & 12 & 9 & 12 & 10 & 8 & 19 & 11 \\
\hline
\end{tabular}

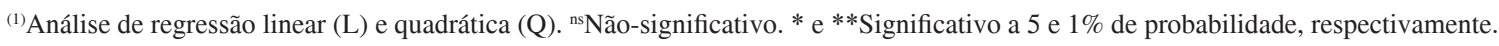



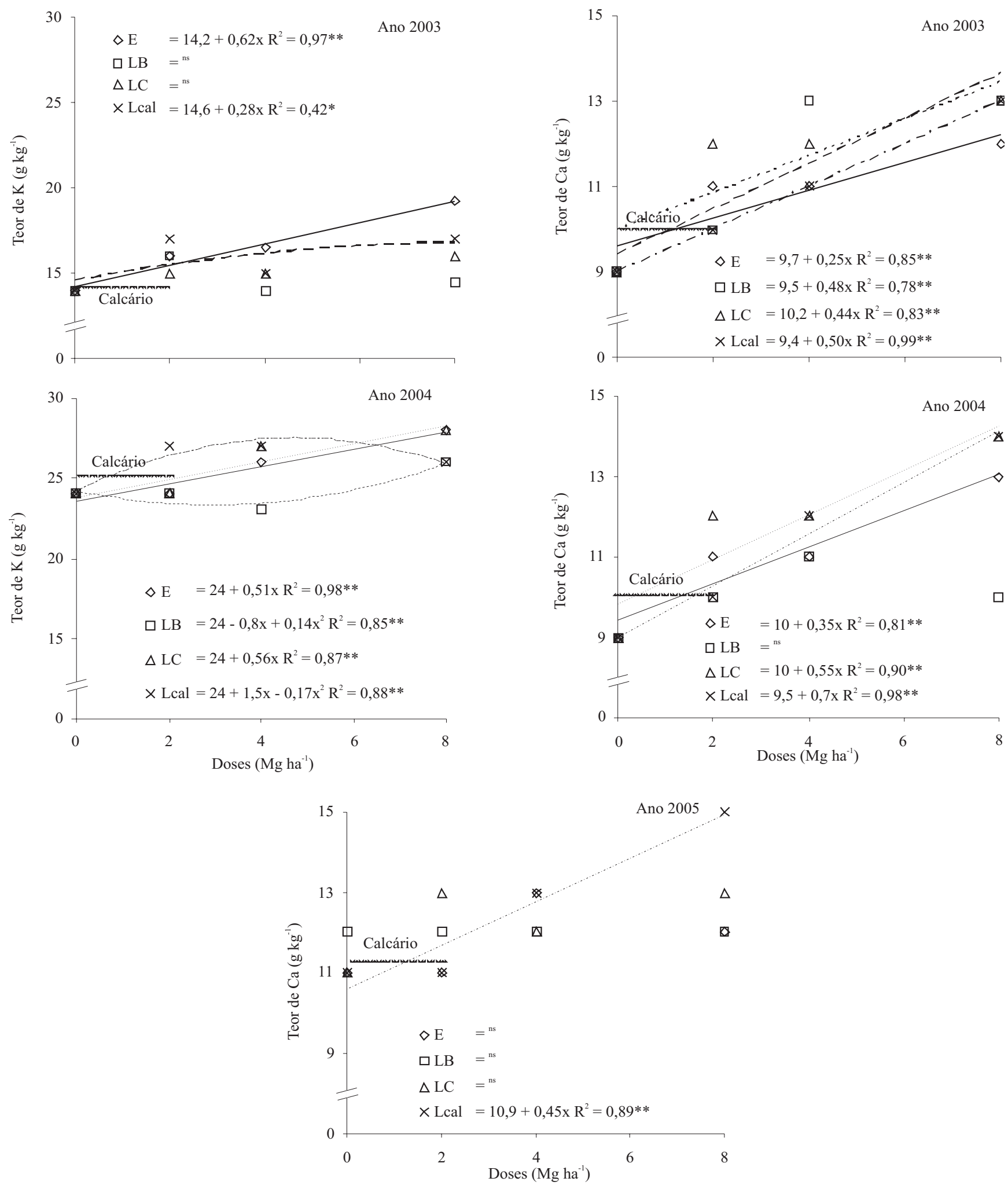

Figura 2. Teores de potássio e cálcio nas folhas de soja, nos anos de 2003, 2004 e 2005 em razão da aplicação de resíduos de lodo de esgoto centrifugado (LC) e de biodigestor (LB), escória de aciaria (E), lama cal (Lcal) e calcário na superfície do solo em sistema plantio direto. Não houve efeito significativo no teor de Ca em 2005. 

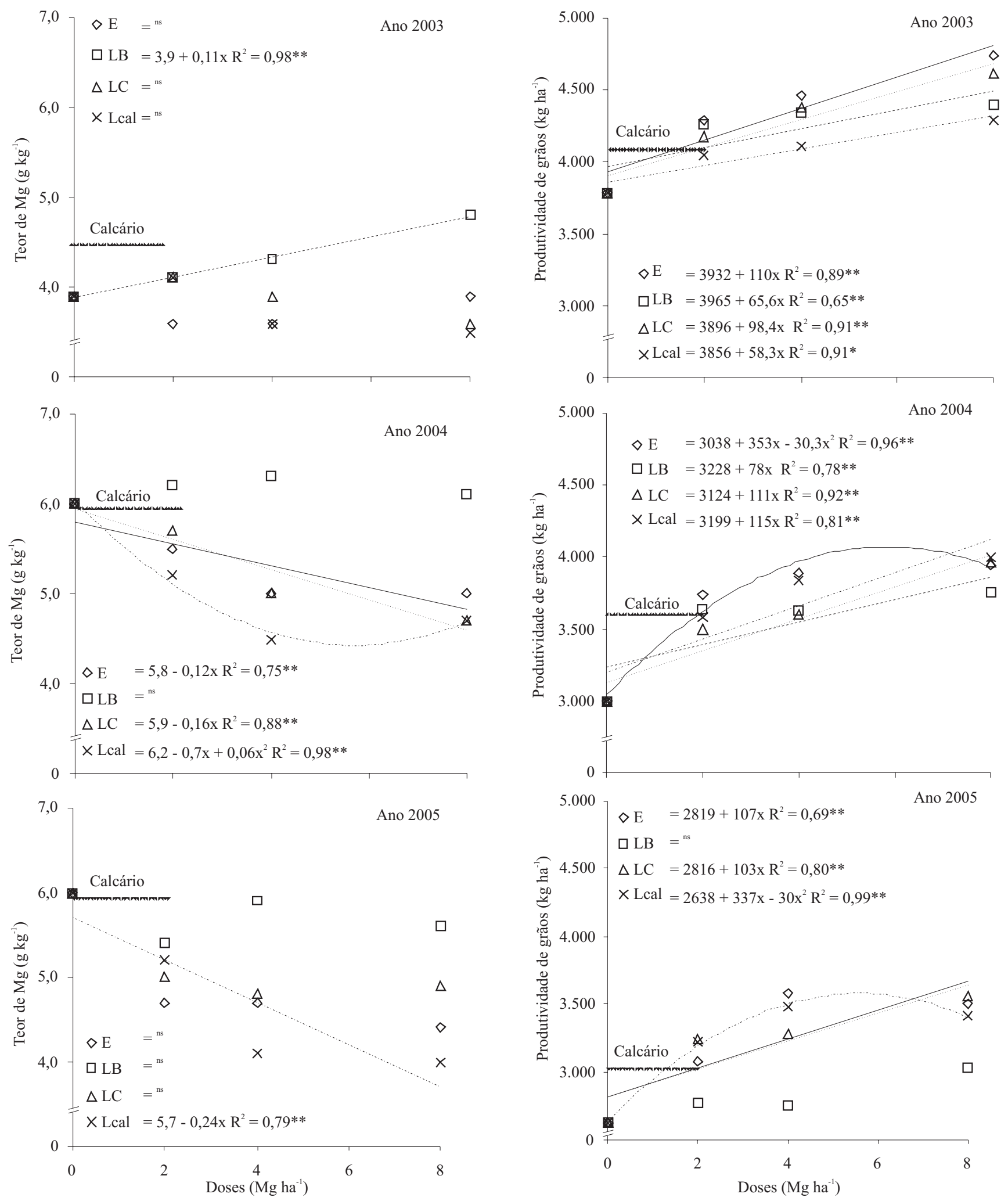

Figura 3. Teor de magnésio e produtividade de grão de soja, nos anos de 2003, 2004 e 2005 em razão da aplicação de resíduos de lodo de esgoto centrifugado (LC) e de biodigestor (LB), escória de aciaria (E), lama cal (Lcal) e calcário na superfície do solo em sistema plantio direto. 
terem fornecido esse elemento de forma direta ao solo, nas quantidades: de 2,24, 0,16, 1,8 e 2,96 $\mathrm{Mg} \mathrm{ha}^{-1}$, respectivamente. No entanto, em 2005, apenas o resíduo Lcal apresentou aumento no teor de Ca na soja, o que pode ser explicado pela provável estabilidade do sistema plantio direto, que proporcionou melhores condições à fertilidade do solo e garantiu o equilíbrio na relação $\mathrm{Ca}, \mathrm{Mg}$ e $\mathrm{K}$. E isso permitiu melhor disponibilidade desse nutriente à soja, e justifica $\mathrm{o}$ alto teor de Ca na folha $\left(11 \mathrm{~g} \mathrm{~kg}^{-1}\right)$ na dose 0 .

De acordo com Malavolta et al. (1999), o teor adequado de Ca para se obter produtividade adequada de soja seria de $10,8 \mathrm{~g} \mathrm{~kg}^{-1}$. Valores próximos ou superiores a esse foram obtidos com a aplicação de resíduos e de calcário.

Houve maior absorção de $\mathrm{Mg}$ pela soja em razão da aplicação de LB nos anos de 2003 e 2004, e redução em razão do aumento das doses do resíduo Lcal no ano de 2005 (Figura 3). Todos os tratamentos apresentaram teores de $\mathrm{Mg}$ na soja próximos ou superiores ao estipulado por Malavolta et al. (1999), de 4,9 $\mathrm{g} \mathrm{kg}^{-1}$, em 2004 e 2005. Já em 2003, os teores encontrados foram inferiores ao estipulado pelo autor, e ficaram próximos de $4 \mathrm{~g} \mathrm{~kg}^{-1}$. Essa redução na absorção de $\mathrm{Mg}$ pela soja está relacionada à maior absorção de $\mathrm{Ca}$, pela planta, para os resíduos $\mathrm{E}$, Lcal e LC (Figura 3), o que confirma o antagonismo na absorção dos nutrientes $\mathrm{Ca}$ e $\mathrm{Mg}$, pelas plantas, com valores de correlação de Pearson entre eles de $\mathrm{R}=-0,81^{* *}$, em 2004, e de $\mathrm{R}=-0,73^{* *}$, em 2005, para os resíduos E, Lcal e LC.

Não houve diferença entre tratamentos para a relação $(\mathrm{Ca}+\mathrm{Mg}) / \mathrm{K}$, sendo a maior relação encontrada de 1,43 no tratamento Lcal, para dose de $8 \mathrm{Mg} \mathrm{ha}^{-1}$, valor aquém do estipulado como limite por Oliveira et al. (2001), que é de 3,6, em que valores superiores a esse, segundo o autor, induzem a sintomas de deficiência de $\mathrm{K}$ na planta, e promove redução no desenvolvimento e na produtividade da soja.

Em 2003, a produção de soja aumentou em até $956 \mathrm{~kg} \mathrm{ha}^{-1}$ com a aplicação superficial dos resíduos e da calagem (Figura 3). Os resíduos que se mostraram mais eficientes para o aumento de produtividade foram escória de aciaria, seguida de $\mathrm{LC}$ e $\mathrm{LB}$ e do Lcal. Houve aumento linear na produção da soja com a dose de resíduo aplicada, o que implica a necessidade de realizar novos estudos com doses superiores, a fim de evidenciar possível ponto de inflexão para a produtividade, desde que utilizados de maneira racional, levando-se em consideração os riscos ambientais, principalmente para os lodos LC e LB, os quais devem ser utilizados de acordo com a Resolução Conama no 375 (Conama, 2006).

$\mathrm{O}$ resultado de produtividade pode ser atribuído à melhoria das propriedades químicas do solo, ao aumento do $\mathrm{pH} \mathrm{em} \mathrm{CaCl}_{2}$, e aos teores de $\mathrm{P}, \mathrm{Ca}$ e $\mathrm{Mg}$ (Tabela 3), fatores que favoreceram à maior absorção dos nutrientes N, P, Ca e K (Figuras 1 e 2).

A produtividade de 2004 foi inferior à de 2003, no entanto, a aplicação superficial dos resíduos e do calcário proporcionou aumento da produção em $996 \mathrm{~kg} \mathrm{ha}^{-1}$, em relação à dose 0 (Figura 3). Assim como em 2003, o resultado de produção em 2004 foi em face da melhoria nos atributos químicos do solo até a profundidade de $20 \mathrm{~cm}$ (Tabela 3), o que permitiu melhor absorção dos nutrientes $\mathrm{N}, \mathrm{P}, \mathrm{K}$ e $\mathrm{Ca}$. Todos resíduos contribuíram para elevar a produtividade em 2004, no entanto, destacam-se os tratamentos Lcal e E na dose de $8 \mathrm{Mg} \mathrm{ha}^{-1}$ e a E, na dose de 5,8 $\mathrm{Mg} \mathrm{ha}^{-1}$. A contribuição do calcário foi intermediária aos tratamentos compostos por resíduos e superior a dose 0 , e contribuiu em acréscimos de mais de $600 \mathrm{~kg} \mathrm{ha}^{-1}$. $\mathrm{O}$ que pode justificar, portanto, o uso desses resíduos, bem como a calagem em superfície sob sistema de plantio direto como alternativa para se obter melhor produtividade de soja.

A aplicação dos resíduos e da calagem proporcionou maior produtividade na cultura da soja, no ano de 2005 (Figura 3), com acréscimo de até $930 \mathrm{~kg} \mathrm{ha}^{-1}$ com a aplicação de LC na dose de $8 \mathrm{Mg} \mathrm{ha}^{-1}$ e de $424 \mathrm{~kg} \mathrm{ha}^{-1}$ para a calagem. No entanto, essa produtividade foi inferior aos anos de 2003 e 2004. O único tratamento que não apresentou interação foi o lodo de esgoto LB. As plantas desenvolvem maior potencial de crescimento em condições de melhor fertilidade, em razão do aumento das doses dos resíduos, bem como do tratamento calcário.

A disponibilidade adequada de $\mathrm{Ca}$ e $\mathrm{Mg}$ trocáveis no solo (Tabela 3) contribuiu para a alta produtividade da soja, corroborando os resultados obtidos por Oliveira \& Pavan (1996) e Caires et al. (2003), que constataram aumento de produção de soja pela aplicação da calagem superficial em solos ácidos. Esses autores atribuem à resposta da cultura à neutralização da acidez do solo na camada superficial e subsuperficial, elevando a saturação por bases e os teores de $\mathrm{Ca}, \mathrm{Mg}$ e $\mathrm{K}$. 
$\mathrm{Na}$ soja, os teores de $\mathrm{P}$ e Ca foram as variáveis que contribuíram para o aumento da produtividade nos anos de 2003 e 2004, e o P teve maior importância que o $\mathrm{Ca}$, em razão da aplicação dos resíduos e da calagem. A regressão linear múltipla entre a produtividade e os macronutrientes absorvidos pela soja em 2003 foi $\mathrm{Y}=1488,6+740,9 \mathrm{P}^{* *}+55,1 \mathrm{Ca}^{* *}$ $\left(\mathrm{R}^{2}=0,63\right)$, e em 2004 foi $\mathrm{Y}=-424,84+974,6 \mathrm{P}^{* *}+$ $69,2 \mathrm{Ca}^{* *}\left(\mathrm{R}^{2}=0,71\right)$. Não houve efeito significativo no ano de 2005, possivelmente por causa da absorção mais equilibrada dos nutrientes, em especial o $\mathrm{P}$ e o $\mathrm{Ca}$, proporcionado pelo sistema plantio direto, assim como ocorrido com o K.

\section{Conclusões}

1. A aplicação de escória de aciaria, lodos de esgoto centrifugado e de biodigestor, lama cal e calagem resulta em maiores concentrações foliares dos nutrientes $\mathrm{N}, \mathrm{P}$, $\mathrm{Ca}$ e K na planta de soja.

2. A produtividade da soja é favorecida pela aplicação superficial de escória de aciaria, lodo de esgoto centrifugado e de biodigestor, lama cal e calagem no sistema plantio direto.

\section{Agradecimentos}

À Fundação de Amparo à Pesquisa do Estado de São Paulo, pela bolsa concedida e pelo apoio financeiro.

\section{Referências}

ABNT. Associação Brasileira de Normas Técnicas. NBR 10004: resíduos sólidos: classificação. Rio de Janeiro, 1987. 63p.

ABREU, M.F.; ABREU, C.A.; ANDRADE, J.C. Determinação de fósforo, potássio, cálcio, magnésio, enxofre, cobre, ferro, manganês, zinco, níquel, cádmio, cromo e chumbo em ácido nítrico usando métodos da US-EPA. In: RAIJ, B. van; ANDRADE, J.C.; CANTARELlA, H.; QUAGGIO, J.A. (Ed.). Análise química para avaliação da fertilidade de solos tropicais. Campinas: Instituto Agronômico, 2001. p.251-261.

ALCÂNTARA, M.A.K. de; AQUINO NETO, V.; CAMARGO. O.A. de; CANTARELLA, H. Mineralização do nitrogênio em solos tratados com lodos de curtume. Pesquisa Agropecuária Brasileira, v.42, p.547-555, 2007.

BANZATTO, D.A.; KRONKA, S.N. Experimentação agrícola. Jaboticabal: Funep, 1989. 247p.

BERTON, R.S.; CAMARGO, O.A.; VALADARES, J.M.A.S. Absorção de nutrientes pelo milho em resposta à adição de lodo de esgoto a cinco solos paulistas. Revista Brasileira de Ciência do Solo, v.13, p.187-192, 1989.
BROWN, S.; ANGLE, J.S.; CHANEY, R.L. Correction of limed-biosolid induced manganese deficiency on a long-term field experiment. Journal of Environmental Quality, v.26, p.1375-1384, 1997.

CAIRES, E.F.; BLUM, J.; BARTH, G.; GARBUIO, F.G.; KUSMAM, M.T. Alterações químicas do solo e respostas da soja ao calcário e gesso aplicados na implantação no sistema de plantio direto. Revista Brasileira de Ciência do Solo, v.27, p.275-286, 2003.

CANTARELLA, H.; QUAGGIO, J.A.; RAIJ, B. van. Determinação da matéria orgânica. In: RAIJ, B. van; ANDRADE, J.C. de; CANTARELlA, H.; QUAGGIO, J.A. (Ed.). Análise química para avaliação da fertilidade de solos tropicais. Campinas: Instituto Agronômico, 2001. p.173-180.

CARVALHO-PUPATTO, J.G.; BÜLL, L.T.; CRUSCIOL, C.A.C. Atributos químicos do solo, crescimento radicular e produtividade do arroz de acordo com a aplicação de escórias. Pesquisa Agropecuária Brasileira, v.39, p.1213-1218, 2004.

CONAMA. Conselho Nacional do Meio Ambiente. Resolução no 375 , de 29 de agosto de 2006. Define critérios e procedimentos, para o uso agrícola de lodos de esgoto gerados em estações de tratamento de esgoto sanitário e seus produtos derivados, e dá outras providências. Diário Oficial [da] República Federativa do Brasil, Brasília, DF, 30 ago. 2006. Disponível em: $<$ http://www.mma.gov.br/port/conama/legiabre. cfm?codlegi=506/normas_01.htm>. Acesso em: 6 ago. 2008.

CORRÊA, J.C.; BÜLL, L.T.; CRUSCIOL, C.A.C.; MARCELINO, R.; MAUAD, M. Correção da acidez e mobilidade de íons em Latossolo com aplicação superficial de escória, lama cal, lodos de esgoto e calcário. Pesquisa Agropecuária Brasileira, v.42, p.1307-1317, 2007.

CORRÊA, J.C.; BÜLL, L.T.; PAGANINI, W.S.; GUERRINI, I.A. Disponibilidade de metais pesados em Latossolo com aplicação superficial de escória, lama cal, lodos de esgoto e calcário. Pesquisa Agropecuária Brasileira, v.43, p.411-419, 2008.

EMBRAPA. Sistema brasileiro de classificação de solos. Brasília: Embrapa Produção de Informação; Rio de Janeiro: Embrapa Solos. 1999. 412p.

FERREIRA, D.F. Sisvar: versão 4.2. Lavras: UFLA, 2003.

LANARV. Laboratório Nacional de Referência Vegetal. Análise de corretivos, fertilizantes e inoculantes: métodos oficiais. Brasília: Ministério da Agricultura, 1983. 104p.

LEMAINSKI, J.; SILVA, J.E. da. Avaliação agronômica e econômica da aplicação de biossólido na produção de soja. Pesquisa Agropecuária Brasileira, v.41, p.1477-1484, 2006.

MALAVOLTA, E. Potássio: absorção, transporte e redistribuição na planta. Informações Agronômicas, v.108, p.1-16, 2004. (Encarte Técnico).

MALAVOLTA, E.; OLIVEIRA, S.A.; WADT, P.G.S. Foliar diagnosis: the status of the art. In: SIQUEIRA, J.O.; MOREIRA, F.M.S.; LOPES, A.S.; GUILHERME, L.R.G.; FAQUIN, V.; FURTINI NETO, A.E.; CARVALHO, J.G. (Ed.). Inter-relação fertilidade, biologia do solo e nutrição de plantas. Viçosa: SBCS, 1999. p.205-242. 
MALAVOLTA, E.; VITTI, G.C.; OLIVEIRA, S.A. Avaliação do estado nutricional de plantas: princípios e aplicações. 2.ed. Piracicaba: Potafos, 1997. 319p.

NASCIMENTO, C.W.A.; BARROS, D.A.S.; MELO, E.E.C.; OLIVEIRA, A.B. Alterações químicas em solos e crescimento de milho e feijoeiro após aplicação de lodo de esgoto. Revista Brasileira de Ciência do Solo, v.28, p.385-392, 2004.

OLIVEIRA, E.L. de; PAVAN, M.A. Control of soil acidity in no-tillage system for soybean production. Soil and Tillage Research, v.38, p.47-57, 1996.

OLIVEIRA, F.A. de; CARMELLO, Q.A. de C.; MASCARENHAS, H.A.A. Disponibilidade de potássio e suas relações com cálcio e magnésio em soja cultivada em casa-de-vegetação. Scientia Agricola, v.58, p.329-335, 2001.

OLIVEIRA, F.C.; MATTIAZZO, M.E.; MARCIANO, C.R.; ROSSETO, R. Efeito de aplicações sucessivas de lodo de esgoto em um Latossolo Amarelo distrófico cultivado com cana-de-açúcar: carbono orgânico, condutividade elétrica, pH e CTC. Revista Brasileira de Ciência do Solo, v.26, p.505-519, 2002.

QUAGGIO, J.A.; RAIJ. B. van. Determinação do pH em cloreto de cálcio e da acidez total. In: RAIJ, B. van; ANDRADE, J.C.; CANTARElla, H.; QUAGGIO, J.A. Análise química para avaliação da fertilidade de solos tropicais. Campinas: Instituto Agronômico, 2001. p.181-188.

RAIJ, B. van; CANTARELLA, H.; QUAGGIO, J.A.; FURLANI, A.M.C. Recomendações de adubação e calagem para o Estado de São Paulo. 2ed. Campinas: Instituto Agronômico; Fundação IAC, 1996. 285p. (Boletim Técnico, 100).

RAIJ, B. van; QUAGGIO, J.A. Determinação de fósforo, cálcio, magnésio e potássio extraídos com resina trocadora de íons. In: RAIJ, B. van; ANDRADE, J.C.; CANTARELLA, H.; QUAGGIO, J.A. Análise química para avaliação da fertilidade de solos tropicais. Campinas: Instituto Agronômico, 2001. p.189-199.

ROSOLEM, C.A.; SILVA, R.H.; ESTEVES, J.A.F. Potassium supply to cotton roots as affected by potassium fertilization and liming. Pesquisa Agropecuária Brasileira, v.38, p.635-641, 2003.

ROSSETTO, C.A.V.; FERNANDES, D.M.; ISHIMURA, I.; ROSOLEM, C.A. Diferentes respostas de cultivares de soja ao potássio. Pesquisa Agropecuária Brasileira, v.30, p.1225-1231, 1995.

SYSTAT SOFTWARE. Systat: programas sigmaplot e sigmastat. Disponível em: <http://www.systat.com>. Acesso em:12 ago. 2008.

VIEIRA, R.F. Sewage sludge effects on soybean growth and nitrogen fixation. Biology and Fertility of Soils, v.34, p.196-200, 2001.

VIEIRA, R.F.; TANAKA, R.T.; TSAI, S.M.; PÉREZ, D.V.; SILVA, C.M.M. de S. Disponibilidade de nutrientes no solo, qualidade de grãos e produtividade da soja em solo adubado com lodo de esgoto. Pesquisa Agropecuária Brasileira, v.40, p.919-926, 2005.

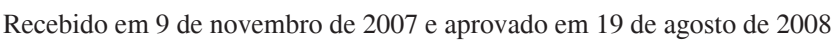


